\title{
SIMPLEXWISE LINEAR UNTANGLING
}

\author{
DAVID W. HENDERSON
}

\begin{abstract}
In this paper we show how to canonically untangle simplexwise linear spanning arcs of a convex 2-cell. Specifically, we show that the space of such arcs is contractible. The main step in the contraction is a flow along the gradient field of an energy function. A 3-dimensional version of this result would imply the Smale Conjecture-Hatcher Theorem.
\end{abstract}

0. Introduction. We will show how to canonically untangle simplexwise linear arcs in the plane. Specifically, let $K^{2}$ be a triangulated convex 2-cell in $\mathbf{R}^{2}$. Let $v$ and $w$ be any two points that lie in the interior of distinct 1-simplexes in the boundary of $K^{2}$. Let $J_{k}$ be the (straight) segment joining $v$ to $w$ and subdivided into $k$ subintervals. Let $\mathscr{T}\left(J_{k}, K^{2}\right)$ denote the space of all maps $f: J_{k} \rightarrow K^{2}$, which are simplexwise linear (i.e. linear on each 1-simplex of $J_{k}$ ) and are such that

$$
f(v)=v, \quad f(w)=w, \text { and } f\left(J_{k}\right) \cap \partial K^{2}=\{v\} \cup\{w\},
$$

where $\partial$ denotes the boundary. Since $f \in \mathscr{T}\left(J_{k}, K^{2}\right)$ is determined by its values on the vertices $v_{1}, v_{2}, \ldots, v_{k-1}$ of $I_{k}$, we can identify $\mathscr{T}\left(J_{k}, I^{2}\right)$ with an (open) subset of $\left(\mathbf{R}^{2}\right)^{k-1}$, where $f$ is identified with the $(k-1)$-tuple $\left(f\left(v_{1}\right), f\left(v_{2}\right), \ldots, f\left(v_{k-1}\right)\right)$. We shall prove

THEOREM. $\mathscr{T}\left(J_{k}, K^{2}\right)$ is contractible.

This is related to the main result of $[\mathbf{B C H}]$ which proves that the space of simplexwise linear homeomorphisms of $K^{2}$, rel $\partial K^{2}$, is contractible. See [BCH] and [CHHS] for a discussion of history, results and questions concerning spaces of simplexwise maps. The main interest in the Theorem and its proof is that it gives a method for attempting a proof of the 3-dimensional version:

CONJECTURE. If $A$ is a polyhedral spanning arc of $I^{3}$ and $\mathscr{T}\left(A, I^{3}\right)$ is the space of simplexwise linear unknotted embeddings of $A$ into $I^{3}$, $\operatorname{rel} \partial I^{3}$, then $\mathscr{T}\left(A, I^{3}\right)$ is contractible.

See $\$ 6$ for a discussion of this conjecture. According to a result in [Hen], this conjecture, if true, would imply the analogous result in the smooth category. This later result is known to be equivalent to the well-known Smale Conjecture, recently proved by A. Hatcher (see [Hat2]).

Received by the editors March 31, 1985 and, in revised form, November 4, 1985.

The author was partially supported by NSF Grant MCS 83-01865. Early stages of this research were done while the author was a visiting faculty member at Birzeit University, West Bank.

1980 Mathematics Subject Classification. Primary 58D10, 57Q35; Secondary 578M99.

(C)1986 American Mathematical Society $0002-9947 / 86 \$ 1.00+\$ .25$ per page 
1. Reduction of the Theorem. If $P$ is a projective transformation of the extended plane which maps at most one point of $K$ to infinity and if $f \in \mathscr{J}\left(J_{k}, K^{2}\right)$, then define $f_{P}: P\left(J_{k}\right) \rightarrow P(K)$ by setting $f_{P}(z)=P \circ f \circ P^{-1}(z)$ for $z$ a vertex of $J_{k}$, and extending linearly. Note that $f_{P} \in \mathscr{T}\left(P\left(J_{k}\right), P\left(K^{2}\right)\right)$ and $f_{P}\left(P\left(J_{k}\right)\right)=$ $P \circ f \circ P^{-1}\left(P\left(J_{k}\right)\right)$, but $f_{P} \neq P \circ f \circ P^{-1}$, since $P$ is not linear on simplexes. Now pick a projective transformation $P$ so that $P\left(J_{k}\right)=[0, k] \times\{0\}$ and so that the $P$-images of the 1-simplexes containing the endpoints of $J_{k}$ are vertical and contain the segments $\{0\} \times[-1,1]$ and $\{k\} \times[-1,1]\left(\subset \mathbf{R}^{1} \times \mathbf{R}^{1}\right)$ (see Figure 1$)$. Note that if $v$ and $w$ belong to adjacent edges of $\partial K$, then $P$ will send the vertex between $v$ and $w$ to $\infty$.

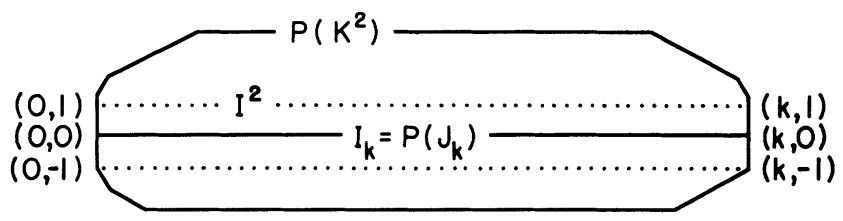

\section{FIGURE 1}

(1.1) LeMmA. $\mathscr{T}\left(J_{k}, K^{2}\right)$ is homeomorphic to $\mathscr{T}\left(P\left(J_{k}\right), P\left(K^{2}\right)\right)$ which contracts within itself into the subspace $\mathscr{T} \equiv \mathscr{T}\left(I_{k}, I^{2}\right)$, where $I_{k} \equiv P\left(J_{k}\right)=[0, k] \times\{0\}$ and $I^{2} \equiv[0, k] \times[-1,1]$

PROOF. It is easy to check that $f \rightarrow f_{P}$ is a homeomorphism with inverse $g \rightarrow g_{P^{-1}}$. The contraction is accomplished by composing each function in $\mathscr{T}\left(P\left(J_{k}\right), P\left(K^{2}\right)\right)$ with the homotopy of $\mathbf{R}^{1} \times \mathbf{R}^{1}$, which takes

$$
(x, y) \mapsto(x,(1-t+t d) y)
$$

at time $t$ for $d=\max \left\{\|y\| \mid y \text { is the } y \text {-coordinate of some point in } P\left(K^{2}\right)\right\}^{-1}$.

In the remainder of the paper we will prove the Theorem by showing that $\mathscr{T} \equiv \mathscr{T}\left(I_{k}, I^{2}\right)$ is contractible.

2. The contraction of $\mathscr{T}$. In order to contract $\mathscr{T}$ it is sufficient to show that each compact subset $\mathscr{C} \subset \mathscr{T}$ can be contracted in $\mathscr{T}$. For $f \in \mathscr{C}$, we proceed by straightening out $f\left(I_{k}\right)$ one vertex at a time. That is, if

$$
\mathscr{T}_{j} \equiv\{f \in \mathscr{T} \mid f(i)=i \text { for } i=1,2, \ldots, j\},
$$

then we homotope $\mathscr{C}$ into $\mathscr{T}_{1}$ and then homotope (in $\mathscr{T}_{1}$ ) the (compact) image of $\mathscr{C}$ into $\mathscr{T}_{2}$, and so forth, until we reach $\mathscr{T}_{k-1}$, which consists only of the identity map. The homotopy of a compact subset of $\mathscr{T}_{j}$ (in $\mathscr{T}_{j}$ ) is accomplished in two steps:

(2.1) Lemma. Any compact subset of $\mathscr{T}_{j}$ is homotopic (in $\mathscr{T}_{j}$ ) to a (compact) subset of

$$
\mathscr{T}_{j}^{*} \equiv\left\{f \in \mathscr{T}_{j} \mid \pi_{x} f(j+1)=j+1\right\}
$$

where $\pi_{x}$ is the projection of $\mathbf{R}^{1} \times \mathbf{R}^{1}$ onto $\mathbf{R}^{1} \times\{0\}$.

(2.2) Lemma. Any compact subset of $\mathscr{T}_{j}^{*}$ is homotopic (in $\left.\mathscr{T}_{j}^{*}\right)$ to a (compact) subset of $\mathscr{T}_{j+1}$. 
Proof of Lemma (2.1) [AND Lemma (2.2)]. The construction of the homotopy is very similar in both steps: In the main text the homotopy of (2.1) will be detailed [the changes necessary for (2.2) will be indicated in square brackets]. The homotopy is defined to be horizontal [vertical]; i.e. $\pi_{y} f_{t}(i)\left[\pi_{x} f_{t}(i)\right]$ will be constant. With these restrictions the homotopy is defined as the flow along the gradient vector field of an energy function $E: \mathscr{T}_{j} \rightarrow[0, \infty)\left[E: \mathscr{T}_{j}^{*} \rightarrow[0, \infty)\right]$. That is, the homotopy $f \mapsto f_{t}$ is defined by letting $f_{t}$ be the solution of the system of equations

$$
\frac{d}{d t} f_{t}(i)=\frac{-\partial}{\partial x(i)} E\left(f_{t}\right) \quad\left[=\frac{-\partial}{\partial y(i)} E\left(f_{t}\right)\right], \quad f_{0}=f,
$$

where $f_{t}(i)=(x(i), y(i))$. Part of $E$ is a "spring energy", $S$, acting as if there were a spring pulling $f(j+1)$ to where it is supposed to go. That is, for (2.1), define $S$ on $\mathscr{T}_{j}$ by setting $S(f)=\left\|\{j+1\}-\pi_{x} f(j+1)\right\|^{2}$. [For (2.2), define $S$ on $\mathscr{T}_{j}^{*}$ by setting $S(f)=\|\{j+1\}-f(j+1)\|^{2}$.] If this were all of $E$, then in most cases during the flow one of the vertices $f_{t}(i)$ would intersect the boundary, $\partial I^{2}$, of $I^{2}$ or some $f_{t}([r, r+1])$ for $r \neq i-1, i$. To prevent this from happening, some "bumper energies", $B$, are added, enabling points and segments to be kept apart. In particular, let $h:(0, \infty) \rightarrow[0, \infty)$ be defined by

$$
h(x)= \begin{cases}\frac{A}{x} \exp \left(\frac{-1}{d-x}\right) & \text { for } 0<x<d, \\ 0 & \text { for } d \leqslant x\end{cases}
$$

which is a monotone decreasing $C^{\infty}$ function depending on the two parameters $A$ and $d$, which will be defined in $\S 4$. Then, if $p$ is a point in the interior of $I^{2}$ and $Q$ is $\partial I^{2}$ or a line segment disjoint from $p$, define $B(p, Q) \equiv h(D(p, Q))$, where $D(p, Q) \equiv \inf \{\|p-q\| q \in Q\}$ is the distance from $p$ to $Q$. Notice that $B(p, Q)$ is zero if $p$ and $Q$ are further apart than $d$ and that, as $p$ approaches $Q, B(p, Q) \rightarrow \infty$. We can now define

$$
E(f) \equiv S(f)+\sum B(p, Q)
$$

where the sum is over all $p=f(i)$ and $Q=\left\{\partial I^{2}\right.$ or $f([r, r+1])$ for $\left.r \neq i-1, i\right\}$. For $f \in \mathscr{T}_{j}$, the first $j$ vertices of $I_{k}$ are fixed and thus we can represent $f=$ $(f(j+1), f(j+2), \ldots, f(k+1)) \in\left(\mathbf{R}^{2}\right)^{k-1-j}$ and $\mathscr{T}_{j}$ is an open subset of $\left(\mathbf{R}^{2}\right)^{k-1-j}$ and $\mathscr{T}_{j}^{*}$ is an open subset of $(\{j+1\} \times \mathbf{R}) \times\left(\mathbf{R}^{2}\right)^{k-2-j}$. Define $f_{t}$ to be the unique solution of the system of differential equations

$$
\frac{d}{d t} f_{t}(i)=\frac{-\partial}{\partial x(i)} E\left(f_{t}\right), \quad f_{0}=f \quad\left[\frac{d}{d t} f_{t}(i)=\frac{-\partial}{\partial y(i)} E\left(f_{t}\right), f_{0}=f\right]
$$

for $j+1 \leqslant i \leqslant k-1$. The theory of ordinary differential equations applies because in $\S 3$ we will show that $\partial E / \partial x(i)[\partial E / \partial y(i)]$ are Lipschitz functions of $f \in \mathscr{T}_{j}$ $\left[\mathscr{T}_{j}^{*}\right]$. In order for $f_{t}$ to move outside of $\mathscr{T}_{j}\left[\mathscr{T}_{j}^{*}\right]$ it is necessary that either $f_{t}$ fails to be one-to-one or $f_{t}(i)$ intersects $\partial I^{2}$ for some $i=j+1, j+2, \ldots, k-1$, but in either case some $B\left(f_{t}(i), Q\right)$ will be infinite, which is impossible since the energy $E\left(f_{t}\right)$ starts finite and, for $z=x[z=y]$,

$$
\frac{d}{d t} E\left(f_{t}\right)=\nabla E\left(f_{t}\right) \cdot \frac{d}{d t} f_{t}=-\sum_{i=j+1}^{k-1}\left\|\frac{\partial}{\partial z(i)} E\left(f_{t}\right)\right\|^{2}<0 .
$$


Thus, if $f$ is in the compact $\mathscr{C} \subset \mathscr{T}_{j}\left[\mathscr{T}_{j}{ }^{*}\right]$, then $f_{t}$ stays in the compact $E^{-1} E(\mathscr{C}) \subset$ $\mathscr{T}_{j}\left[\mathscr{T}_{j}^{*}\right]$. It follows from the standard theory of differential equations (see, for example, [Spi, pp. 194-200] that $(f, t) \rightarrow f_{t}$ defines a continuous flow on $\mathscr{T}_{j}\left[\mathscr{T}_{j}^{*}\right]$ for $t \in[0, \infty)$. Let

$$
\nabla_{z} E \equiv\left(\frac{\partial}{\partial z(j+1)} E, \ldots, \frac{\partial}{\partial z(k-1)}\right) \text { for } z=x[z=y]
$$

In $\S 5$ we will show

(2.3) Lemma. $S(f) \neq 0$ implies that $\nabla_{z} E(f) \neq 0$ for $z=x[z=y]$.

Then we conclude that, for all $S\left(f_{t}\right)>\varepsilon$, there is a $\delta$ such that

$$
\frac{d}{d t} E\left(f_{t}\right)=\nabla_{z} E\left(f_{t}\right) \cdot \frac{d}{d t} f_{t}=-\left\|\nabla_{z} E\left(f_{t}\right)\right\|^{2}<\delta<0,
$$

and thus $\lim _{t \rightarrow \infty} S\left(f_{t}\right)=0$, since $E\left(f_{t}\right)$ is bounded below by 0 . Now, we do not know that this flow can be extended to $t=\infty$; however, eventually $f_{t}(j+1)$ will be so close to its destination that we can stop the flow (for all $j+1 \leqslant i \leqslant k-1$ simultaneously) and continue the homotopy by moving only $f(j+1)$ along the straight line to its destination, $\left(j+1, \pi_{y} f(j+1)\right)[(j+1,0)]$. This finishes the proof of (2.1) [(2.2)].

3. $\partial E / \partial x(i)$ and $\partial E / \partial x(i)$ are Lipschitz functions. Since

$$
\frac{\partial}{\partial z} E(f)=\frac{\partial}{\partial z} S(f)+\sum h^{\prime}(D(p, Q)) \frac{\partial}{\partial z} D(p, Q)
$$

for $z=x(i)[y(i)]$, it suffices to show that $(\partial / \partial z) D(p, Q)$ is Lipschitz for $p=f(s)$ and $Q=\left\{\partial I^{2}\right.$ or $\left.f([r, r+1]), r \neq s-1, s\right\}$. We see that if $p=f(s)$ and $Q=$ $f([r, r+1])$, then

$$
\nabla_{i} D \equiv\left(\frac{\partial}{\partial x(i)} D(p, Q), \frac{\partial}{\partial x(i)} D(p, Q)\right)
$$

is nonzero only if $i=s, r$ or $r+1$. We use the fact that the gradient, $\nabla_{i} D$, is in the direction that $f(i)$ must be moved to obtain the maximum rate of increase of $D$ and that that rate is the magnitude of $\nabla_{i} D$. Let $q$ be the closest point of $Q$ to $p$. We have three cases: $q=f(r), q=f(r+1)$, or $q \in f((r, r+1))$.

(i) $q=f(r)$. Then $D(p, Q)=\|f(s)-f(r)\|$ and

$$
\nabla_{s} D=\frac{f(s)-f(r)}{\|f(s)-f(r)\|}=-\nabla_{r} D \quad \text { and } \quad \nabla_{r+1} D=0 \text {. }
$$

(ii) $q=f(r+1)$. Then $D(p, Q)=\|f(s)-f(r+1)\|$ and

$$
\nabla_{s} D=\frac{f(s)-f(r+1)}{\|f(s)-f(r+1)\|}=-\nabla_{r+1} D \quad \text { and } \quad \nabla_{r} D=0 .
$$

(iii) $q \in f((r, r+1))$. Then, if $\mu$ is the unit vector perpendicular to $Q$ and pointing towards $p$, we have

$$
\begin{gathered}
\nabla_{s} D=\mu, \quad \nabla_{r} D=\frac{-\|q-f(r+1)\|}{\|f(r)-f(r+1)\|} \cdot \mu, \quad \text { and } \\
\nabla_{r+1} D=\frac{-\|q-f(r)\|}{\|f(r)-f(r+1)\|} \cdot \mu .
\end{gathered}
$$


We notice that these gradients are continuous, but not differentiable. But restricted to each of the three pieces each gradient is differentiable and bounded (in $\mathscr{T})$. Thus these gradients are Lipschitz.

4. The constants $c, A, d$, and the orderings $<$ and $o$. Throughout this section $\mathscr{C}$ is some compact subset of $\mathscr{T}_{j}\left[\mathscr{T}_{j}^{*}\right]$. Notice that the homotopy of $\mathscr{T}_{j}\left[\mathscr{T}_{j}^{*}\right]$ into $\mathscr{T}_{j}^{*}$ $\left[\mathscr{T}_{j+1}\right]$ will be contained in the set $\mathscr{C}^{\sim}, \mathscr{C} \subset \mathscr{C}^{\sim} \subset \mathscr{T}_{j}\left[\mathscr{T}_{j}{ }^{*}\right]$, where we set $g \in \mathscr{C}^{\sim}$ iff there is an $f \in \mathscr{C}$ such that

(a) $\pi_{y} f(t)=\pi_{y} g(t)\left[\pi_{x}\right]$ for all $t \in I_{k}$.

(b) for all $t, t^{\prime} \in I_{k}, \pi_{y} f(t)=\pi_{y}\left(t^{\prime}\right)\left[\pi_{x}\right]$ implies that $\left\{\pi_{x} f(t), \pi_{x} f\left(t^{\prime}\right)\right\}$ has the same order as $\left\{\pi_{x} g(t), \pi_{x} g\left(t^{\prime}\right)\right\}\left[\pi_{y}\right]$.

(c) $E(g) \leqslant E(\mathscr{C}) \equiv \operatorname{Max}\left\{E\left(f^{\prime}\right) \mid f^{\prime} \in \mathscr{C}\right\}$.

(4.1) Lemma. There is a constant $c \equiv c(\mathscr{C})<\frac{1}{4}$ such that, for all $1 \leqslant r, s \leqslant k-1$ and $g \in \mathscr{C}^{-}$, whenever the horizontal [vertical] line through $g(s)$ intersects $g([r-1, r])$ and $g([r, r+1])$ on opposite sides of $g(s)$, then $\left\|\pi_{y} g(s)-\pi_{y} g(r)\right\| \geqslant c$ $\left[\left\|\pi_{x} g(s)-\pi_{x} g(r)\right\| \geqslant c\right]$.

Proof. We shall prove (4.1) only for the case of $\mathscr{T}_{j}$ and leave to the reader the modifications necessary for $\mathscr{T}_{j}^{*}$. Suppose that $\left\{g_{i}\right\} \subset \mathscr{C}^{\sim}$ such that the horizontal line through $g_{i}(s)$ intersects $g_{i}([r-1, r])$ at $p_{i}$ and intersects $g_{i}([r, r+1])$ at $q_{i}$ on opposite sides of $g(s)$ and suppose that

$$
\left\|\pi_{y} g_{i}(s)-\pi_{y} g_{i}(r)\right\| \rightarrow 0 .
$$

Then, for the corresponding $f_{i} \in \mathscr{C}$, some subsequence of $\left\{f_{i}\right\}$ will converge to $f \in \mathscr{C}$, since $\mathscr{C}$ is compact. Thus

$$
\lim \pi_{y}\left(p_{i}\right)=\lim \pi_{y}\left(q_{i}\right)=\pi_{y} f(s)=\pi_{y} f(r)
$$

and $\pi_{x} f(s)$ is between $\lim \pi_{x}\left(p_{i}\right)$ and $\lim \pi_{x}\left(q_{i}\right)$. Therefore, $f(s) \in f([r-1, r+1])$, which is impossible since $f \in \mathscr{T}$ is one-to-one.

(4.2) Definitions. (i) Set $b \equiv b(\mathscr{C}) \equiv 2(k / c)^{2}>2 k / c>8 k$.

(ii) Choose $d=d(\mathscr{C})>0$ so small that $b^{2 k} d \leqslant c / 2$.

(iv) Pick $A=A(\mathscr{C})$ so large that $h(x) \leqslant E(\mathscr{C})$ implies $\frac{3}{4} d \leqslant x$.

For what follows, assume that $f \in \mathscr{C}^{\sim}$. We now use the above defined constants to construct several orderings on the segments: $P_{r}=f([r, r+1]), 0 \leqslant r \leqslant k-1$.

(4.3) Definition. (a) $P_{i}<{ }_{a} P_{r}$ iff there is a horizontal [vertical] line in $I^{2}$ which first (traveling from left to right [down to up]) intersects $P_{i}$ and then intersects $P_{r}$.

(b) $P_{i}<{ }_{\mathrm{b}} P_{r}$ iff $P_{i}<{ }_{\mathrm{a}} P_{r_{1}}<{ }_{\mathrm{a}} P_{r_{2}}<{ }_{\mathrm{a}} \cdots<{ }_{\mathrm{a}} P_{r_{n}}<{ }_{\mathrm{a}} P_{r}$, for some $n$.

(c) $P_{i}<{ }_{\mathrm{c}} P_{r}$ iff $P_{r}$ not- $<_{\mathrm{b}} P_{i}$ and the projections $\pi_{y} P_{i}\left[\pi_{x} P_{i}\right]$ and $\pi_{y} P_{r}\left[\pi_{x} P_{r}\right]$ are disjoint and, for all those endpoints, $p \in P_{i}$ and $q \in P_{r}$, whose $\pi_{y}\left[\pi_{x}\right]$-images are closest, it is true that these images are within $d$ of each other (see (4.2)), and $\pi_{x} p<\pi_{x} q\left[\pi_{y}\right]$ or $\left\{\pi_{y}\left(\pi_{y} \mid P_{i}\right)^{-1} \pi_{y} p \cap \pi_{x}\left(\pi_{y} \mid P_{r}\right)^{-1} \pi_{y} q\right.$ is nonempty and $\left.\pi_{y} p<\pi_{y} q\right\}$ [interchange $\pi_{x}$ and $\pi_{y}$ ].

(d) $P_{i}<P_{r}$ iff $P_{i}<\mathbf{e}_{0} P_{r_{1}}<e_{\mathbf{e}_{1}} P_{r_{2}}<\mathbf{e}_{2} \cdots<\mathbf{e}_{n-1} P_{r_{n}}<{ }_{\mathbf{e}_{n}} P_{r}$ for some $n$, where for each $i, \mathbf{e}_{i}=\mathbf{a}$ or $\mathbf{e}_{i}=\mathbf{c}$. 
Notice that $<_{\mathbf{c}}$ is discontinuous with respect to $f$ in the sense that, for some $f$, $P_{i}<_{\mathrm{c}} P_{r}$ but, for some $g$ arbitrarily near $f, P_{r}<_{\mathrm{c}} P_{i}$. This is the main source of the $g_{f}$ constructed in $\S 5$ being discontinuous if $f$. We now prove

(4.4) Lemma. It is not true that $P_{r}<P_{r}$. Thus $<$ is a partial ordering on the $\left\{P_{i}\right\}$ for each $f \in \mathscr{C}^{-}$.

Proof. Suppose $P_{r_{1}}<P_{r_{2}}<\cdots<P_{r_{n}}$ are adjacent elements in the ordering, i.e., for each $i$, there is no $P_{s}$ such that $P_{r_{i}} \leqslant P_{s} \leqslant P_{r_{i-1}}$. It follows that, for each $1 \leqslant i \leqslant n-1, P_{r_{i}} \leqslant \mathbf{e}_{i} P_{r_{i+1}}$, where $\mathbf{e}_{i}=\mathbf{a}$ or $\mathbf{e}_{i}=\mathbf{c}$. Let $p_{i} \in P_{r_{i}}$ and $q_{i+1} \in P_{r_{i+1}}$ be such that $\pi_{y}\left(p_{i}\right) \pi_{y}\left(q_{i+1}\right)\left[\pi_{y}\right]$ if $\mathbf{e}_{i}=\mathbf{a}$, or $\left\|\pi_{y}\left(p_{i}-q_{i+1}\right)\right\|<a\left[\pi_{x}\right]$ if $\mathbf{e}_{i}=\mathbf{c}$. Look at the piecewise-linear path

$$
\Lambda \equiv p_{1}-q_{2}-p_{2}-q_{3}-p_{3}-\cdots-q_{n-1}-p_{n-1}-q_{n} .
$$

At each vertex of $\Lambda$ whose $y$ [x]-coordinate is a local extremum the path turns towards the right [top] and thus the first (in traversing $\Lambda$ from $p_{1}$ to $q_{n}$ ) point $p \in \Lambda$, if any, which intersects a point on the path before $p$ must be as in Figure 4 .
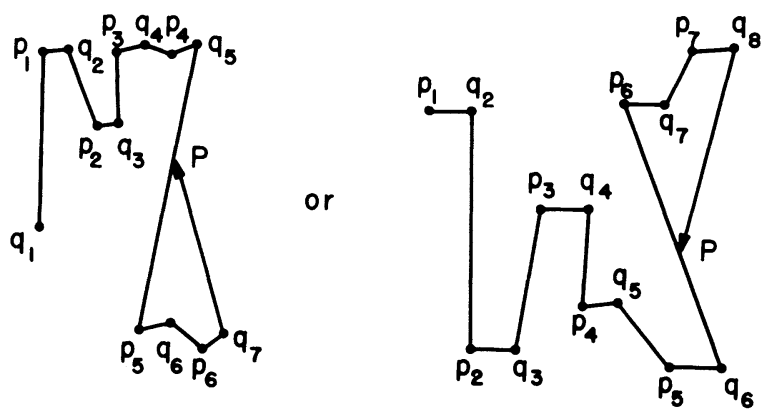

FIGURE 4

If there is such a $p$ then there are four possibilities: For some $r<s$, (i) $p=\left[q_{r}, p_{r}\right]$ $\cap\left[q_{s}, p_{s}\right]$, (ii) $p=\left[q_{r}, p_{r}\right] \cap\left[p_{s}, q_{s+1}\right]$, (iii) $p=\left[p_{r}, q_{r+1}\right] \cap\left[q_{s}, p_{s}\right]$, or (iv) $p=$ $\left[p_{r}, q_{r+1}\right] \cap\left[p_{s}, q_{s+1}\right]$. Since $\left[q_{i}, p_{i}\right] \subset P_{r_{i}}$, if (i) were true then we would have $P_{r_{r}}=P_{r_{s}} \equiv P$ and from Figure 4 we see that $p_{r}, q_{r}$ must be in the same order on $P$ as $q_{s}, p_{s}$ and thus either $p_{r} \in\left[q_{s}, p_{s}\right]$ or $q_{s} \in\left[p_{r}, q_{r}\right]$. However, both of these are impossible since $q_{r+1}$ is left of [below] $p_{r}$ and $p_{s-1}$ is right of [above] $q_{s}$ and $p$ is the first point of intersection; thus we conclude that (i) is impossible. If $\mathbf{e}_{s}=\mathbf{a}$ then (ii) is impossible since $p_{s}$ and $p_{s+1}$ are assumed to be adjacent in the ordering. If $\mathbf{e}_{s}=\mathbf{c}$ then (ii) would imply that $P_{r_{r}}$ is less than $\frac{3}{4} d$ from either $P_{r_{s}}$ or $P_{r_{s+1}}$, which is not possible by (4.2). Similarly, (iii) is impossible. From Figure 4 we see that at least one of the intervals of $\Lambda$ containing $p$ must point towards the left [bottom], thus (iv) is impossible.

In the construction of $g_{f}$ in $\S 5$, we shall use the above partial ordering of the $P_{i}$ and a related total ordering induced by a bijection:

(4.5) Definition. Choose $o:\{0,1, \ldots, k-1\} \rightarrow\{0,1, \ldots, k-1\}$ to be a one-toone function so that:

(a) If $o(r)<o(s)$, then either $P_{r}<P_{s}$, or $P_{r}$ and $P_{s}$ are incomparable. 
(b) If no segment $P_{i} \in f\left(I_{k}\right)$ is between (in the < -partial ordering) two adjacent segments $\left\{P_{r-1}, P_{r}\right\}$, then $o(r-1)=o(r)+1$, or $o(r)=o(r+1)+1$.

(c) If $S \equiv\left\{P_{0}, P_{1}, \ldots, P_{s}\right\}$ is maximal with respect to satisfying (b) for each pair $\left\{P_{r}, P_{r+1}\right\}, 0 \leqslant r<s$, then $o(i)<o(0)$ iff $P_{i}<P_{r}$ for some $r \leqslant s$. (Note that $j \leqslant s$.

Define $m(i) \equiv \min \{o(i), o(i-1)\}$ and $M(i) \equiv \operatorname{Max}\{o(i), o(i-1)\}$. Note that $f(i)$ belongs to $P_{i-1}$ and $P_{i}$, and so $m(i)$ gives the first place and $M(i)$ the last place that $f(i)$ is represented in the ordering $o$.

(4.6) Lemma. Let $f \in \mathscr{C}^{\sim}$. If $\pi_{y} f(i)$ is within $d$ of $\pi_{y}\left(P_{r-1} \cup P_{r}\right)\left[\pi_{x}\right]$ and $\pi_{y} f(i)$ $\left[\pi_{x}\right]$ is within $c=c(\mathscr{C})$ of $\pi_{y} f(r)\left[\pi_{x}\right]$, then either $m(i) \geqslant M(r)$ or $M(i) \leqslant m(r)$, with equality only if $i=r \pm 1$.

Proof. By (4.1) it is impossible that $f(i)$ be between $P_{r}$ and $P_{r-1}$. Therefore, either (a) $\pi_{y} f(i)$ intersects both $\pi_{y} P_{r}$ and $\pi_{y} P_{r-1}\left[\pi_{x}\right]$, (b) $\pi_{y} P_{r} \cap \pi_{y} P_{r-1}=\pi_{y} f(r)$ [ $\pi_{x}$ ], or (c) $\pi_{P r}$ or $\pi_{y} P_{r-1}$ is contained in $\left[\pi_{y} f(i), \pi_{y} f(r)\right]\left[\pi_{x}\right]$. Thus, by (4.1) and (4.5)(b), we see in each case that neither $P_{i-1}$ nor $P_{i}$ can come between $P_{r}$ and $P_{r-1}$ in the $>$-ordering. Therefore the desired inequalities hold with equality only if $P_{r}=P_{i-1}$ or $P_{r-1}=P_{i}$.

5. Proof of Lemma (2.3). Preparatory to proving (2.3) at the end of this section, we construct a certain function $g_{f} \in \mathscr{C}^{\sim}$ for each $f \in \mathscr{C}^{\sim}$.

(5.1) LeMma. If $f \in \mathscr{C}^{\sim}$ and $S(f) \neq 0$, then there is a $g_{f} \in \mathscr{C}^{\sim}$ such that:

(a) $\pi_{y} g_{f}(i)=\pi_{y} f(i)\left[\pi_{x}\right], 0 \leqslant i \leqslant k$,

(b) for all $0 \leqslant s \leqslant 1$, sf $+(1-s) g_{f} \in \mathscr{T}_{j}\left[\mathscr{T}_{j}^{*}\right]$,

(c) $E\left(g_{f}\right)=S\left(g_{f}\right)=0$,

(d) $\left(f(j+1)-g_{f}(j+1)\right) \cdot \nabla_{j+1} S(f)>0$,

(e) for all $0 \leqslant s \leqslant 1, B\left(s f+(1-s) g_{f}\right)<B(f)$, where $B(\cdot)$ ranges over all $B=B(p, Q)$ that appear in the definition of $E(\cdot)$.

We will prove (5.1) after giving the inductive construction of $g_{f}$ in (5.2). The $g_{f}$ which we construct will not vary continuously with $f$; in fact, note that if we could find the $g_{f}$ that varied continuously with $f$, then (5.1) would produce the desired homotopy of $\mathscr{T}_{j}\left[\mathscr{T}_{j}^{*}\right]$.

In order to construct $g_{f}$ we make use of the constants $d, b, c$, and the orderings $<, \quad o$ of $\$ 4$. Recall $m(i) \equiv \min \{o(i), o(i-1)\}$ and $M(i) \equiv$ $\operatorname{Max}\{o(i), o(i-1)\}$, and let $r \equiv o^{-1}:\{0,1, \ldots, k-1\} \rightarrow\{0,1, \ldots, k-1\}$. We define $g_{f}$ inductively, one segment at a time. In the case that $\pi_{x} f(j+1)>j+1$ $\left[\pi_{v} f(j+1)>0\right]$, we define $g_{f}$ first on $[r(0), r(0)+1]$ and then on $[r(1), r(1)+1]$ and so on in order until $[r(k-1), r(k-1)+1]$. In the case that $\pi_{x} f(j+1)<j+1$ $\left[\pi_{y} f(j+1)<0\right]$, we define $g_{f}$ on the segments in the reverse order. We shall describe in detail only the first case; the changes necessary for the second case should be clear to the reader. 
$(5.2)_{s}$ LEMMA. In the case that $\pi_{x} f(j+1)>j+1\left[\pi_{y}(j+1)>0\right]$, the function $g_{f}$ can be defined on

$$
C s \equiv \bigcup_{i=0}^{s-1}[r(i), r(i)+1]
$$

so that the following hold:

(a) $g_{f}$ is linear on each interval $[i, i+1] \in C s, \pi_{y} g_{f}(p)=\pi_{y} f(p)\left[\pi_{x} g_{f}(p)=\right.$ $\left.\pi_{x} f(p)\right]$, and, for $0 \leqslant i \leqslant j, g_{f}(i)=(i, 0)$ and $g_{f}(k+1)=(k+1,0)$, whenever defined.

(b) s There is a finite subset

$$
T s \equiv\left\{g_{f}(i) \mid M(i) \geqslant s\right\} \cup\left\{p_{1}, p_{2}\right\} \subset I^{2},
$$

where $p_{1}=\left(b^{2 k} d, \operatorname{sign}\left(\pi_{y} f(j+1)\right) 1\right)\left[=\left(0,-1+b^{2 k} d\right)\right]$ and $p_{2}=\left(b^{2 k} d, 0\right) \quad[=$ $\left.\left(k,-1+b^{2 k} d\right)\right]$ if $s \leqslant o(0)$, and $p_{1}=\left(j+1+b^{2 k} d,-\right)\left[=\left(0, b^{2 k} d\right)\right]$ and $p_{2}=(j$ $\left.+1+b^{2 k} d, 1\right)\left[=\left(k, b^{2 k} d\right)\right]$ if $s>o(0)$. For $p \in T s$, define $M(p)=M(i)$ if $p=$ $g_{f}(i)$, and $M\left(p_{1}\right)=M\left(p_{2}\right)=o(0)$ if $s \leqslant o(0)$, and $M\left(p_{1}\right)=M\left(p_{2}\right)=k$ if $s>o(0)$. We order $T s$ by the order on $\pi_{y} T s\left[\pi_{x} T s\right]$. Except for $p_{1}, p_{2}$, Ts is just those vertices of $g_{f}(C s)$ which are endpoints of only one simplex in $g_{f}(C s)$.

(c) $)_{s}$ Let $q, q^{\prime}$ be in Ts. If, for every $r$ between $q$ and $q^{\prime}$ in $T s, M(r)<M(q)$ and $M(r)<M\left(q^{\prime}\right)$ and if $\left[q, q^{\prime}\right]$ is not contained in Cs, then, for all $0<\tau<1$, either (i) $p \equiv \tau \cdot q+(1-\tau) \cdot q^{\prime}$ is at least as far horizontally [vertically] as

$$
\begin{aligned}
D\left(q, q^{\prime} ; \tau ; s\right) & \equiv\left(\tau b^{2 M(q)}+(1-\tau) b^{2 M\left(q^{\prime}\right)}\right) d\left(b^{-1}-s b^{-2}\right) \\
& =\tau\left(b^{2 M(q)-1}-s b^{2 M(q)-2}\right) d+(1-\tau)\left(b^{2 M(q)-1}-s b^{2 M(q)-2}\right) d
\end{aligned}
$$

from any point within $d$ of Cs or (ii) $\pi_{y} p\left[\pi_{x} p\right]$ is within $c$ of $\pi_{y} q\left(\right.$ or $\left.\pi_{y} q^{\prime}\right)\left[\pi_{x}\right]$ and $p$ is closer horizontally [vertically] than $D\left(q, q^{\prime} ; \tau ; s\right)$ to the $g_{f}$-image of some interval containaing $q$ (or $\left.q^{\prime}\right)$, where $M(p) \equiv \tau \cdot M(q)+(1-\tau) \cdot M(q)$ (see Figure 5.1).

(d) $)_{s}$ Let $q \in T s$. If $M(q)<o(0)$ and $p \in J q$ with $\pi_{y} p=\pi_{y} f(j+1)\left[\pi_{x}\right]$, then $(j+1)-\pi_{x} p\left[0-\pi_{y} p\right] \geqslant b^{2 k} d$. If $M(q)>o(0)$ and $q \in T s$, then (c) holds for $q^{\prime} \equiv\left(j+1+b^{2 k} d, \pm 1\right)\left[\equiv\left(0, b^{2 k} d\right)\right.$ or $\left.\left(k, b^{2 k} d\right)\right]$ with $M\left(q^{\prime}\right) \equiv k$.

(e) $)_{s}$ If $m(i) \leqslant o(0) \leqslant M(i)$, then $j+1 \leqslant \pi_{x} g_{f}(i) \leqslant j+1+b^{2 k} d\left[0 \leqslant \pi_{y} g_{f}(i) \leqslant\right.$ $\left.b^{2 k} d\right]$.

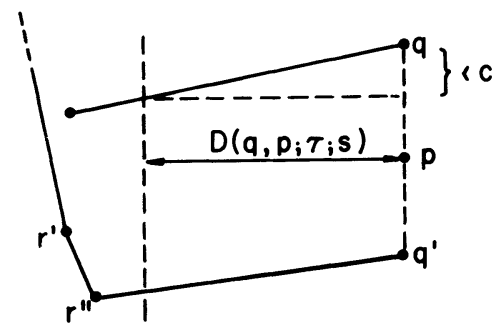

Figure 5.1 
Proof That $(5.2)_{s}$ Implies $(5.2)_{s+1}$. We add $[r(s), r(s)+1]$ to $C s$ and have three cases depending on whether both, neither, or only one of $r(s), r(s)+1$ are in Cs.

Both $r(s)$ and $r(s)+1$ are in Cs. In this case $q=q_{f}(r(s))$ and $q^{\prime}=g_{f}(r(s)+1)$ are in $T s$. If $\pi_{y} g_{f}(i)$ is between $\pi_{y} q\left[\pi_{x} q\right]$ and $\pi_{y} q^{\prime}\left[\pi_{x} q^{\prime}\right]$, then $M(i)<s=M(s)$ and therefore $q_{f}(i) \notin T s$. Thus $q$ and $q^{\prime}$ are adjacent (in the $\pi_{y}\left[\pi_{x}\right]$-induced order on $T s$ ) and, by (c) $)_{s}$, we can extend $g_{f}$ linearly on $[r(s), r(s)+1]$ and it will still be one-to-one and will satisfy (a) $)_{s+1}$. The two points on either side of $q$ and $q^{\prime}$ in $T s$ are now adjacent in $T s+1$. The other parts of $(5.2)_{s+1}$ now follow if you note that, for each $q \in T s, s<M(q)$ and thus that $b^{2 s} \leqslant b^{2 M(q)-2}$ (see Figure 5.2).

Only one of $r(s)$ and $r(s)+1$ are in Cs. Say $r(s)$ is not in $C s$ and $r(s)+1$ is in $C s$ (and $\left.q=g_{f}(r(s)+1) \in T s\right)$. By the same argument as above, no $r \in T s$ can be between $q$ and $l \equiv \pi_{y}^{-1} \pi_{y}(r(s))\left[\equiv \pi_{s}^{-1} \pi_{x}(r(s))\right]$ in the ordering induced by $\pi_{y}\left[\pi_{x}\right]$. Thus $l$ must be between $q$ and some $p \in T s$ which is adjacent to $q$ in $T s$. Note that $M(q)=s$ and so all other $p \in T s$ have larger $M$ and thus will be in $T s+1$. Let $q_{1}$ and $q_{2}$ be the first points in $T s$ on each side of $l$ such that $M\left(q_{1}\right), M\left(q_{2}\right)>M(r(s))$, if there are any such. If on a side of $l$ there is no such point $q_{1}$, then note that $M(r(s)) \geqslant M\left(p_{i}\right) \equiv o(0)$ and set

$$
q_{1}=\left(j+1+b^{2 k} d, \pm 1\right) \quad\left[=\left(0, b^{2 k} d\right) \text { or }\left(k, b^{2 k} d\right)\right] .
$$

If $\pi_{y} l=\tau \pi_{y} q_{1}+(1-\tau) \pi_{y} q_{2}\left[\pi_{x}\right]$, then set $q^{\prime \prime} \equiv \tau q_{1}+(1-\tau) q_{2}$ and define

$$
\pi_{x} g_{f}(r(s))=\pi_{x} q^{\prime \prime}-D\left(q_{1}, q_{2} ; \tau ; s\right)+b^{2 M(r(s))} d \quad\left[\pi_{y}\right]
$$

Now define $q^{\prime} \equiv g_{f}(r(s))$ and thus $M\left(q^{\prime}\right)=M(r(s))$. Extend linearly to $[r(s), r(s)$ $+1]$ (see Figure 5.3).

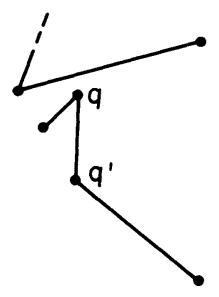

Figure 5.2

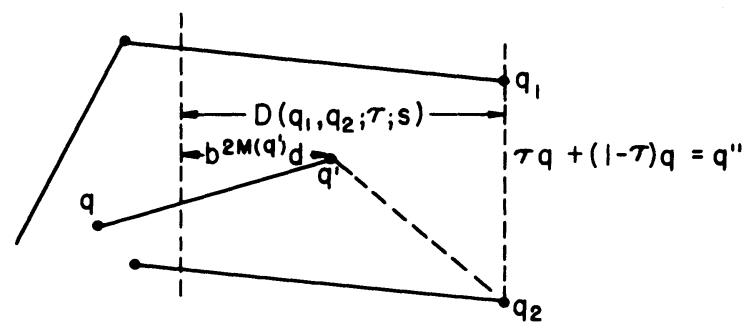

FIGURE 5.3 


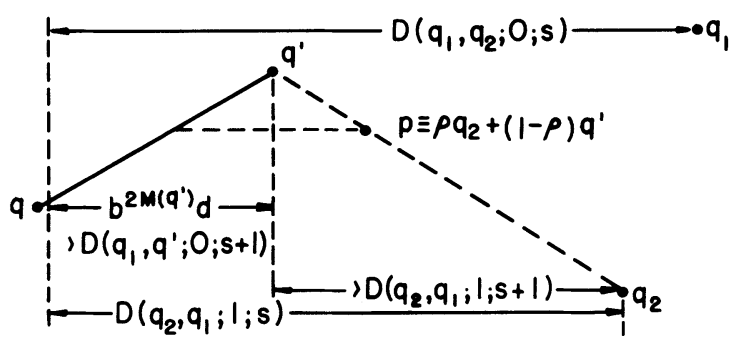

Figure 5.4

If (c) $)_{s}$ (ii) holds for $q_{1}, q_{2} ; \tau$, then by (4.6) we conclude $M\left(q^{\prime}\right) \leqslant m\left(q_{1}\right) \quad$ or $\left.M\left(q^{\prime}\right) \leqslant m\left(q_{2}\right)\right\}$ which is impossible; thus we conclude that $q^{\prime \prime}$ is at least as far horizontally [vertically] from $C s$ as $D\left(q_{1}, q_{2} ; \tau ; s\right)$. Thus $q^{\prime}$ misses $C s$ and (see Figure 5.3)

$$
\begin{aligned}
\left\|q^{\prime}-q^{\prime \prime}\right\| & =D\left(q_{1}, q_{2} ; \tau ; s\right)-b^{2 M\left(q^{\prime}\right)} d \\
& =\left\{\tau b^{2 M\left(q_{1}\right)}+(1-\tau) b^{2 M\left(q_{2}\right)}\right\}\left(b^{-1}-s b^{-2}\right) d-b^{2 M\left(q^{\prime}\right)} d \\
& >D\left(q_{1}, q_{2} ; \tau ; s+1\right)
\end{aligned}
$$

since $M\left(q^{\prime}\right) \leqslant M\left(q_{i}\right)-1$.

We must now check $(\mathrm{c})_{s+1}$. Let $q^{\sim} \equiv \alpha q_{1}+(1-\alpha) q_{2}$ be such that $\pi_{y}\left(q^{\sim}\right)=$ $\pi_{y}(q)$. Then, by (c) $)_{s}\left\|q-q^{\sim}\right\|>D\left(q_{1}, q_{2} ; \alpha ; s\right)$. Thus, for $\beta q^{\prime}+(1-\beta) q \in$ $g_{f}[r(s), r(s)+1]$, we have

$$
\begin{aligned}
&\left\|\beta q^{\prime}+(1-\beta) q-\left(\beta q^{\prime \prime}+(1-\beta) q^{\sim}\right)\right\|=\beta\left\|q^{\prime}-q^{\prime \prime}\right\|+(1-\beta)\left\|q-q^{\sim}\right\| \\
& \geqslant \beta D\left(q_{1}, q_{2} ; \tau ; s+1\right)+(1-\beta) D\left(q_{1}, q_{2} ; \alpha ; s+1\right) \\
& \geqslant D\left(q_{1}, q_{2} ; \beta \tau+\alpha-\beta \alpha ; s+1\right) .
\end{aligned}
$$

For $\lambda \in[0,1]-[\alpha, \tau]$, the horizontally [vertically] closest point to $\lambda q_{1}+(1-\lambda) q_{2}$ is in Cs. Thus (c) $)_{s+1}$ holds for $q_{1}, q_{2}$.

All other cases of (c) $)_{s+1}$ follow from (c) $)_{s}$ except for $q^{\prime}, q^{*}$, where $\pi_{y} q^{*}$ is between or equal to $\pi_{y} q_{1}$ and $\pi_{y} q_{2}$. (Possibly $q^{*}$ is $q_{1}$ or $q_{2}$.) We first set $q^{*}=q_{2}$ and look at $p=\rho q^{\prime}+(1-\rho) q_{2}, 0 \leqslant \rho \leqslant 1$ (see Figure 5.4). If the horizontal [vertical] distance from $p$ to $\left[q, q^{\prime}\right]$ is less than $D\left(q^{\prime}, q_{2} ; \rho ; s+1\right)$, then, using similar triangles,

$$
\begin{aligned}
\left\|\pi_{y} p-\pi_{y} q^{\prime}\right\| & <\left\{D\left(q_{2}, q^{\prime} ; \rho ; s+1\right)-\rho D\left(q_{2}, q^{\prime} ; 1 ; s+1\right)\right\} \cdot \frac{\left\|\pi_{y} q-\pi_{y} q^{\prime}\right\|}{b^{2 M\left(q^{\prime}\right)} d} \\
& <(1-\rho) b^{2 M\left(q^{\prime}\right)} d\left(b^{-1}-(s+1) b^{-2}\right) \frac{k}{b^{2 M\left(q^{\prime}\right)} d}<\frac{k}{b}<\frac{c}{2}
\end{aligned}
$$

(see Figure 5.4). It is also clear from Figure 5.4 that, if $\pi_{y} p$ is farther than $c$ from $\pi_{y} q^{\prime}$, then $p$ is at least as far horizontally [vertically] as $D\left(q_{2}, q^{\prime} ; \rho ; s+1\right)$ from any point which is within $d$ of $C s+1$.

If remains now to look at (c) $)_{s+1}$ with $q^{\prime}$ and $q^{*} \neq q_{1}, q_{2}$. Suppose that $\pi_{y} q^{*}$ is between $\pi_{y} q^{\prime}$ and $\pi_{y} q_{2}$ and let $q^{\wedge}$ be the first point in Ts on the other side of $q^{\prime}$ from $q^{*}$ such that $M\left(q^{\wedge}\right)>M\left(q^{*}\right)$. Notice that $q^{*}, q^{\wedge}$ satisfy the hypotheses of 


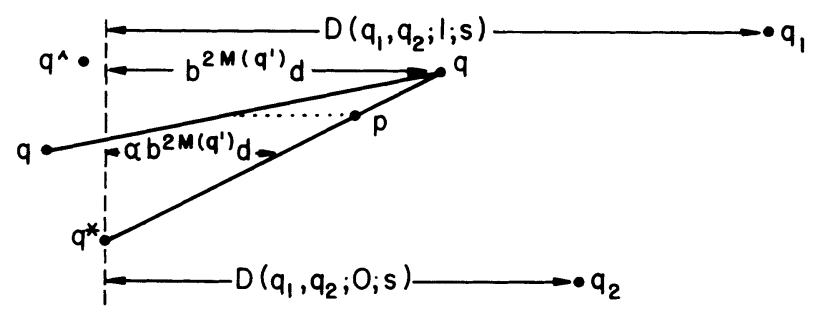

FIGURE 5.5

(c) $)_{s}$. If $q^{\wedge}=q_{1}$, then an argument the same as that for $q^{\prime}, q_{2}$ will show that (c) $)_{s+1}$ is satisfied for $q^{\prime}, q^{*}$. If $q^{\wedge} \neq q_{1}$, then the situation is as in Figure 5.5. Note that by Lemma (4.6), $\left\|\pi_{y} q-\pi_{y} q^{*}\right\|>c$ and thus that

$$
\alpha \equiv \frac{\left\|\pi_{y} q-\pi_{y} q^{*}\right\|}{\left\|\pi_{y} q^{\prime}-\pi_{y} q^{*}\right\|}>\frac{c}{k} .
$$

If $p \equiv \beta q^{*}+(1-\beta) q^{\prime}$ is horizontally [vertically] within $D\left(q^{*}, q^{\prime} ; \beta ; s+1\right)$, then (since $M\left(q^{*}\right)<M\left(q^{\prime}\right)$ )

$$
\begin{aligned}
\left\|\pi_{y} q^{\prime}-\pi_{y} p\right\| & \leqslant D\left(q^{*}, q^{\prime} ; \beta ; s+1\right) \cdot \frac{\left\|\pi_{y} q-\pi_{q}^{\prime}\right\|}{\alpha b^{2 M\left(q^{\prime}\right)} d} \\
& <\left\{\beta b^{2 M\left(q^{*}\right)-2 M\left(q^{\prime}\right)}+(1-\beta)\right\} \cdot \frac{k}{b} \cdot \frac{k}{c}<\frac{k^{2}}{b c}=\frac{c}{2} .
\end{aligned}
$$

An examination of Figure 5.5 shows that (c) $)_{s+1}$ holds for $q^{\prime}, q^{*}$.

Note that (d) $)_{s+1}$ holds if $M\left(q^{\prime}\right)<o(0)$ because then $q^{\prime}$ is further from $g_{f}(j+1)$ than the line joining $q_{1}$ and $q_{2}$, and neither $q_{1}$ nor $q_{2}$ is $\left(j+1+b^{2 k} d, \pm 1\right)$ $\left[\left(0, b^{2 k} d\right)\right.$ or $\left.\left(k, b^{2 k} d\right)\right]$. If $\left.M\left(q^{\prime}\right)\right)>o(0)$ and there is a $w \in T s$ on the side of $\pi_{y}^{-1} \pi_{y} f(j+1)\left[\pi_{x}\right]$ with $M(w) \geqslant o(0)$, then $\pi_{y}\left[q, q^{\prime}\right]\left[\pi_{x}\left[q, q^{\prime}\right]\right]$ does not contain $\pi_{y} f(j+1)\left[\pi_{x}\right]$. If $M\left(q^{\prime}\right)>o(0)$ and there is a $w \in T s, M(w) \geqslant o(0)$, only on the side of $q^{\prime}$ away from $\pi_{y}^{-1} \pi_{y} f\left((\operatorname{see} j+1)\left[\pi_{x}\right]\right.$, then $w, p_{2}, p=\left[w, p_{2}\right] \cap \pi_{y}^{-1} \pi_{y} f(j+1)$ $\left[\pi_{x}\right]$ satisfy $(\mathrm{c})_{s}$ and $(\mathrm{d})_{s}$ and, since $\left[q, q^{\prime}\right]$ must cross $\left[w, p_{2}\right]$, we see that $\left[q^{\prime}, p_{2}\right]$ must miss $C s+1$ and have slope at least $j\left[1-b^{2 k} d\right]$; but $\left|\pi_{y} f(j+1)-\pi_{y} q^{\prime}\right| \geqslant c$ $\left[\pi_{x}\right]$, and thus

$$
\begin{aligned}
j+1-\pi_{x}\left\{\left[q, q^{\prime}\right] \cap\right. & \left.\pi_{y}^{-1} \pi_{y} f(j+1)\right\} \geqslant c j \geqslant b^{2 k} d \\
& {\left[0-\pi_{y}\left\{\left[q, q^{\prime}\right] \cap \pi_{x}^{-1} \pi_{x} f(j+1)\right\} \geqslant c\left(1-b^{2 k} d\right) \geqslant b^{2 k} d\right] }
\end{aligned}
$$

(see (4.2) and Figure 5.6). If there is no $w \in T s, M(w) \geqslant o(0)$, then $\pi_{x} g_{f}(C s)<b^{2 o(0)}$ $\left[\pi_{y}\right]$ and thus $\left[q, p_{2}\right]$ is as in the last sentence and we conclude that $(\mathrm{c})_{s+1}$ and $(\mathrm{d})_{s+1}$ hold.

Neither $r(s)$ nor $r(s)+1$ are in Cs. The proof of this case is essentially the same as the previous case, except that we must pick two pairs of $q_{1}, q_{2}$, one for each of $r(s)$ and $r(s)+1$. 


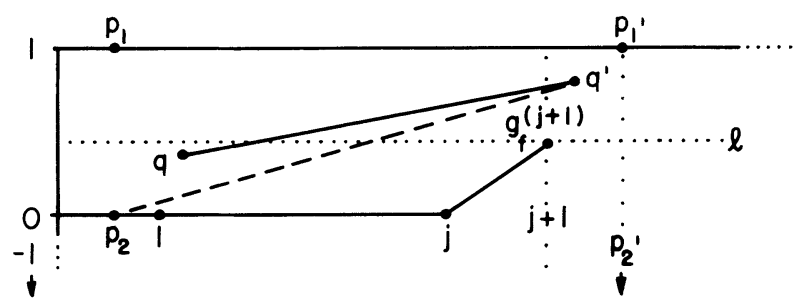

Figure 5.6

PRoof of (5.1). The order $<$ on the segments $P_{r}$ (see (4.3)) ensures that if $B\left(f(i), P_{r}\right) \neq 0$, then $f(i)$ and $P_{r}$ stay in the same order as $g_{f}(i)$ and $g_{f}([r, r+1])$. Since the homotopy is horizontal [vertical] and $D\left(g_{f}(i), g_{f}([r, r+1])\right)>d$, it follows that $B\left(s f+(1-s) g_{f}\right)$ must decrease. (5.1) now follows.

Proof of (2.3). Let $\mu \equiv g_{f}-f \subset\left(\mathbf{R}^{2}\right)^{k-1}$. Then from (5.1) we see that $\mu$. $\nabla_{y} B(f) \leqslant 0\left[\nabla_{x}\right]$ and $\mu \cdot \nabla_{y} S(f)<0\left[\nabla_{x}\right]$. Thus, $\mu \cdot \nabla_{y} E(f)<0\left[\nabla_{x}\right]$ and we conclude that $\nabla_{y} E(f) \neq 0\left[\nabla_{x}\right]$.

6. Comments on 3-dimensional conjecture. Let $A$ be a straight unknotted spanning arc of $I^{3}$ which has a fixed subdivision. Let $\mathscr{T}^{3}$ be the space of simplexwise linear unknotted imbeddings of $A$ into $I^{3}$, rel $\partial I^{3}$. It has been thought (see [Hat1]) that a proof that $\mathscr{T}^{3}$ is contractible is not feasible since there seems to be no way to use the hypothesis of unknottedness in a canonical way. However, it might be possible to define energy functions on $\mathscr{T}^{3}$ and attempt to flow along the gradient vector field in a manner similar to that above in the 2-dimensional case. The unknotted hypothesis would come in when one attempted to prove the analogue of Lemma (2.3), but in this case one would only be looking at a single $f \in \mathscr{T}^{3}$ and one could assume that the image of $f$ bounds a disk in $I^{3}$ and not need to pick the disc canonically. This is analogous to the situation in $\S 5$ where the $g_{f}$ did not depend continuously on $f$.

\section{REFERENCES}

[BCH] E. Bloch, R. Connelly and D. Henderson, The space of simplexwise linear homeomorphisms of a convex 2-disk, Topology 23 (1984), 161-175.

[CHHS] R. Connelly, D. Henderson, C.-w. Ho and M. Starbird, On the problems related to linear homeomorphisms, embeddings, and isotopies, Continua, Decompositions, Manifolds, Univ. of Texas Press, Austin, Texas, 1983, pp. 229-239.

[Hat1] A. Hatcher, Linearization in 3-dimensional topology, Proc. I.C.M. (Helsinki, 1978), Acad. Sci. Fenn., Helsinki, 1980, pp. 463-468.

[Hat2] _ A proof of the Smale Conjecture, Ann. of Math. 117 (1983), 553-607.

[Hen] D. Henderson, Relations between spaces of simplexwise linear and smooth embeddings, Preprint.

[Spi] M. Spivak, A comprehensive introduction to differential geometry, Vol. 1, Publish or Perish, Wilmington, Del., 1979.

Department of Mathematics, Cornell University, Ithaca, New York 14853 